"This document is the Accepted Manuscript version of a Published Work that appeared in final form in NANO LETTERS, copyright (C) American Chemical Society after peer review and technical editing by the publisher. To access the final edited and published work see:

https://doi.org/10.1021/acs.nanolett.5b04174" 


\section{Reversible 2D phase transition driven by an electric field: visualization and control on the atomic scale}

B. Wortmann ${ }^{1}$, D. van Vörden ${ }^{1}$, P. Graf ${ }^{1}$, R. Robles ${ }^{2}$, P. Abufager ${ }^{2,3}$, N. Lorente ${ }^{2,4,5}$, C. A. Bobisch $^{1}$, R. Möller ${ }^{1} *$

${ }^{1}$ Faculty of Physics and Center for Nanointegration Duisburg-Essen (CENIDE), University of Duisburg-Essen, Lotharstr.1-21 47048 Duisburg, Germany

${ }^{2}$ ICN2 - Institut Catala de Nanociencia i Nanotecnologia, Campus UAB, E-08193 Bellaterra (Barcelona), Spain

${ }^{3}$ Instituto de Física de Rosario, Consejo Nacional de Investigaciones Científicas y Técnicas (CONICET) and Universidad Nacional de Rosario, Av. Pellegrini 250 (2000) Rosario, Argentina

${ }^{4}$ Centro de Física de Materiales, CFM/MPC (CSIC-UPV/EHU), Paseo Manuel de Lardizabal 5, 20018 Donostia-San Sebastián, Spain

${ }^{5}$ Donostia International Physics Center (DIPC), Paseo Manuel de Lardizabal 4, 20018

Donostia-San Sebastián, Spain

*Correspondence to: rolf.moeller@uni-due.de

\section{Abstract:}

We report on a reversible structural phase transition of a two dimensional system which can be locally induced by an external electric field. Two different structural configurations may coexist within a $\mathrm{CO}$ monolayer on $\mathrm{Cu}(111)$. The balance between the two phases can be shifted by an external electric field, causing the domain boundaries to move, increasing the area of the favored phase controllable both in location and size. If the field is further enhanced new domains nucleate. The arrangement of the $\mathrm{CO}$ molecules on the $\mathrm{Cu}$ surface is observed in real time and real space with atomic resolution while the electric field driving the phase transition is easily varied over a broad range. Together with the well-known molecular manipulation of $\mathrm{CO}$ adlayers, our findings opens exciting prospects for combining spontaneous long-range order with man-made CO structures such as "molecule cascades" [1] or "molecular graphene" [2]. Our new manipulation mode permits us to bridge the gap between fundamental concepts and the fabrication of arbitrary atomic patterns in large scale, by providing unprecedented insight into the physics of structural phase transitions on the atomic scale. 


\section{Introduction}

Phase transitions are ubiquitous in science from fundamental physics, chemistry or biology to ecological or social systems, and have a broad range of applications, e.g. data storage, drug delivery, etc. [2-7]. Arising from an ensemble of a huge number of microscopic objects, like atoms or molecules, phase transitions manifest themselves as changes of a macroscopic order parameter, like conductivity or magnetization. For a detailed understanding of the different phases as well as the mechanism of phase transitions, knowledge about the individual objects is required. Various techniques based on microscopy or diffraction have been applied to analyze the structure of different phases [8-17].

The arrangement of adsorbates on a surface is governed by interactions between the adsorbate and the atoms of the supporting surface as well as between the adsorbates themselves. The geometric structure, these adsorbates assemble into, can be studied by ensemble averaging techniques like electron diffraction [8] or real space imaging techniques like low electron energy microscopy (LEEM) [9], electron microscopy [10], scanning tunneling microscopy (STM) [11] and atomic force microscopy (AFM) [12]. The latter two allow for the determination of the positions of individual atoms or molecules on the atomic scale.

Moreover, the tip of an STM or AFM may act as a local probe to provide a stimulus to induce phase changes. Despite the finite size attained by individual manipulation of molecules, manmade structures have proven to be of great interest $[1,2]$. The combination of STM-induced manipulations and the long-range ordering of phase transition is a promising route that needs to be explored. 
Phase transitions induced by a change of the temperature have been analyzed by STM or high resolution electron microscopy, for example for $\mathrm{Pb}$ on $\mathrm{Si}(111)$ [13], $\mathrm{Sn}$ on $\mathrm{Si}(111)$ [14], for self-assembled monolayers [15], for roughening transitions of $\mathrm{Au}(110)$ and $\operatorname{Pt}(110)$ [16] or for the semiconducting to metallic phase transition of a $\mathrm{MoS}_{2}$ layer [17]. External electric fields may prompt 3D-phase transitions in ferroelectric materials $[18,19]$ or the freezing of thin layers of water [20,21]. Similarly, the potential in an electrochemical environment or a liquid solid interface can induce transitions between different phases of molecular layers on surfaces $[22,23]$. Furthermore, mechanical strain is predicted to induce a transition, for example from a non-metallic to a metallic phase [24]. Due to short range forces between the apex of the tip and the surface, the tip of an STM or AFM can be used to induce changes of a very small area, e.g. a transition between two different reconstructions of the $\operatorname{Si}(100)$ surface at low temperature $[25,26]$ or a change of the $\mathrm{H}$ induced reconstruction on $\mathrm{Si}(100)$ [27]. However, these modifications do not persist if the tip is retracted from that area of the surface. On the other hand by increasing the bias voltage and the tunneling current of an STM, modification of a phase [28] or nucleation of crystallites of inorganic [29] and organic layers [30] can be induced.

Our experiment reveals a new aspect of adsorbed layers of $\mathrm{CO}$ on $\mathrm{Cu}(111)$. We use the tip of an STM to induce a structural phase transition between two phases of a monolayer of CO on a $\mathrm{Cu}(111)$ surface. As will be shown, the reversible transitions between an $\alpha$-phase $(7 \times 7$ superstructure) and a $\beta$-phase $(3 \sqrt{3} \times 3 \sqrt{3}) \mathrm{R} 30^{\circ}$ are caused by the electric field between the tip and the sample surface. 


\section{Geometric structure of the two phases}

The surface is prepared by dosing $\mathrm{CO}$ in excess onto a clean $\mathrm{Cu}(111)$ surface at $80 \mathrm{~K}$. At saturation, the $\alpha$-phase is formed covering the entire surface. The molecular ordering appears to be stable if the surface is imaged by the STM using a negative bias at the sample. However, if a high enough positive bias is applied for an extended period of time, patches of a new phase, referred to as $\beta$-phase appear and grow continuously. At $80 \mathrm{~K}$, the two phases may coexist on the surface. Fig. 1 displays an STM image with both superstructures separated by an atomic step edge; to the lower left the $7 \times 7$, to the upper right the $3 \sqrt{3} \times 3 \sqrt{3}$. The inset shows the atomically resolved $\mathrm{Cu}(111)$ substrate in the same scaling.

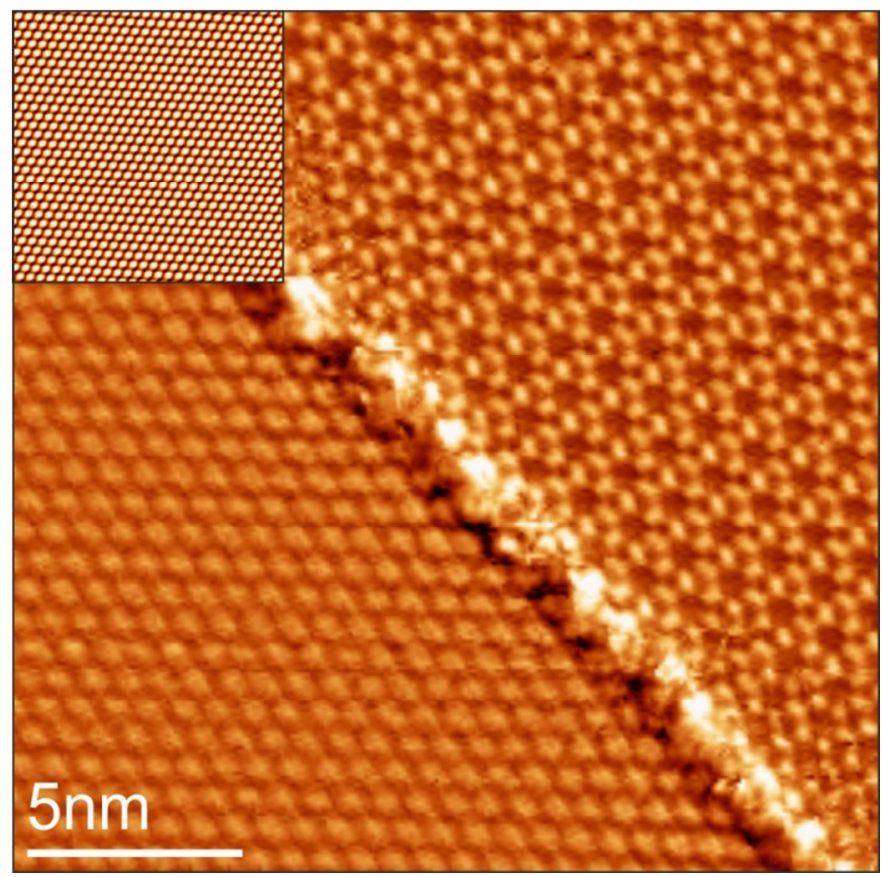

Fig. 1 STM image showing the coexistence of the $\alpha$-phase (lower left) and the $\beta$-phase (upper right) separated by an atomic step of the copper substrate (To enhance the molecular structure in comparison to the height difference of the atomic layers, "unsharp masking" has been applied to the data.). The inset in the upper left corner shows the atomically resolved bare copper surface on the same lateral scale.

Both structures were previously studied by electron diffraction [31]. A $7 \times 7$ superstructure was proposed for the $\alpha$-phase [32] which is now confirmed by our observations. However, the combination of DFT calculations and measured data reveals the individual positions of the 
$\mathrm{CO}$ molecules which differ from the original model. Fig. 2 a-c show the structure and the corresponding model in more detail. The $7 \times 7$ unit cell contains 25 molecules corresponding to a coverage of $\Theta=\frac{25}{49}=0.51 \mathrm{CO}$-molecules per $\mathrm{Cu}$ surface atom. 19 of them are bound to one copper atom of the substrate (top site). With the exception of the molecule in the corner of the unit cell they are all more or less tilted. The 6 molecules marked by blue circles are most likely bound to three of the substrate atoms (threefold hollow site). This corresponds well to measurements by IR absorption and electron loss spectroscopy, predicting two different adsorption sites [31, 33]. As expected from our structural model the contribution attributed to the three fold hollow site (blue circles) is weaker than the one for the top site. 

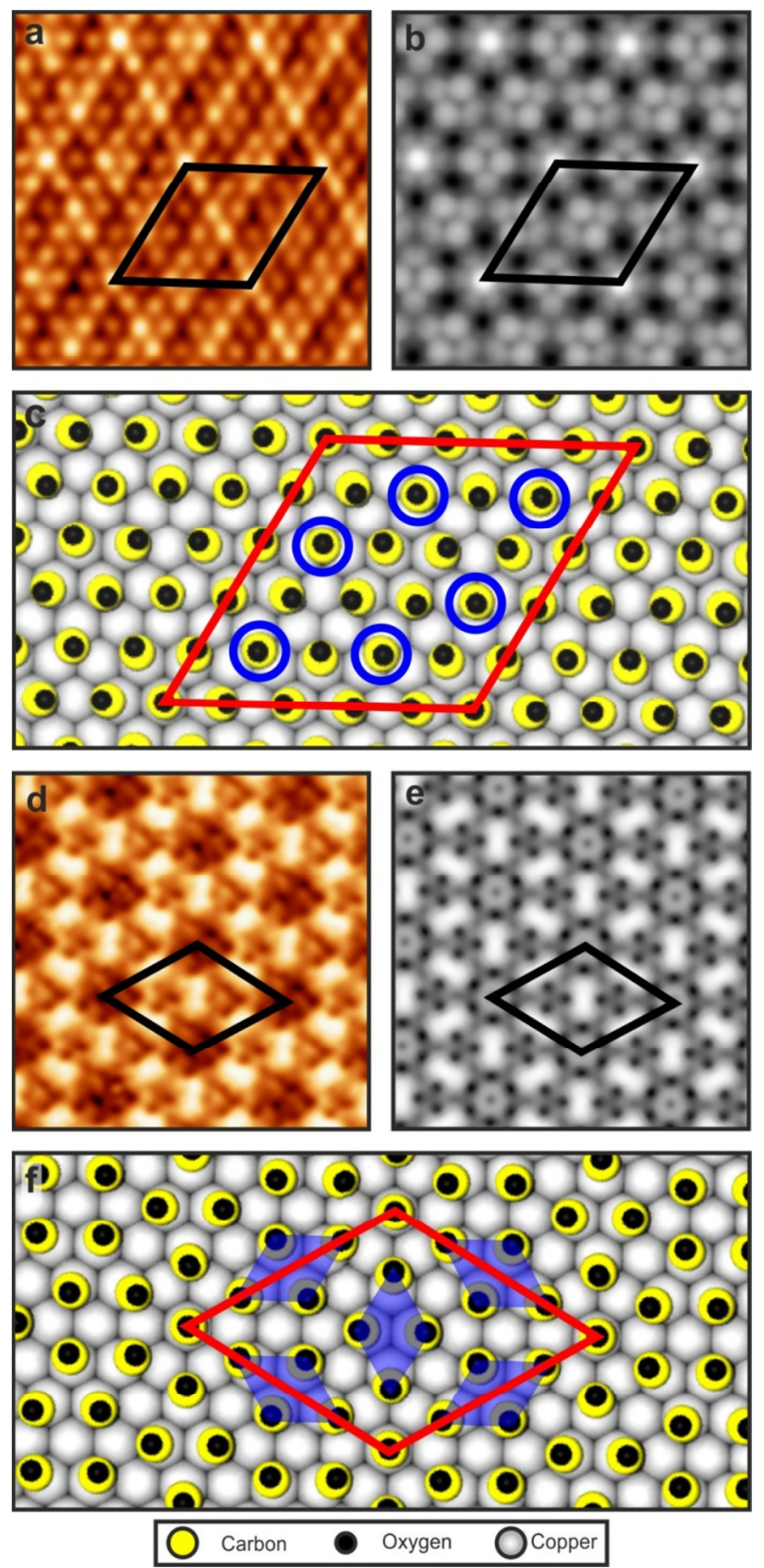

Fig. 2 STM image and model based on DFT calculations for the $(7 \times 7)$ superstructure $(\alpha-$ phase) (a-c) and the $(3 \sqrt{3} \times 3 \sqrt{3})$ R3 $30^{\circ}$ superstructure ( $\beta$-phase) (d-f). a was acquired at a tunneling bias of $-0.4 \mathrm{~V}$ and a tunneling current of $50 \mathrm{pA}$. The area is about $4 \times 4 \mathrm{~nm}^{2} . \mathrm{b}$ shows the corresponding simulated STM image based on the relaxed positions of the CO-molecules in the unit cell displayed in c according to the DFT calculations. Blue circles mark COmolecules not adsorbed on top sites leading to a dim feature. $\mathrm{d}$ was acquired at a tunneling bias of $+0.5 \mathrm{~V}$ and a tunneling current of $50 \mathrm{pA}$. The area is about $4 \times 4 \mathrm{~nm}^{2}$. e shows the corresponding simulated STM image based on the relaxed positions of the CO-molecules in the unit cell displayed in $\mathrm{f}$ according to the DFT calculations. Blue rhombuses mark a building block of the structure leading to a bright feature. 
A careful analysis for the $\beta$-phase based on our STM observations at high resolution reveals a $3 \sqrt{3} \times 3 \sqrt{3}$ unit cell rotated by $30^{\circ}$ with respect to the substrate, instead of the $c(4 \times 2)$ or $1.5 R \times 1.5 R 18^{\circ}$ structures which were originally proposed based on electron diffraction data [34]. Fig. 2 d-f show the structure at higher resolution and its structural model. A building block of this structure is a bright feature in the STM image which is given by an arrangement of $4 \mathrm{CO}$ molecules indicated by the blue rhombuses in the model. It is similar to the “chevrons" formed by $3 \mathrm{CO}$ described by Heinrich et al. [1]. Each unit cells contains three (1 and $4 \times 1 / 2$ ) of these rhombuses plus one $\mathrm{CO}$ at the corner, leading to a coverage of $\Theta=\frac{13}{27}=0.48 \mathrm{CO}$ molecules per $\mathrm{Cu}$ atom.

Since the real space configuration we have found will lead to an almost identical diffraction pattern as observed in [34] and the experimental parameters are very similar, we conclude that the $\beta$-phase is identical to the one previously identified as $1.5 R \times 1.5 R 18^{\circ}$. Our data also correspond well to the experimental results of a previous STM study [35], although a different structural model was proposed.

DFT calculations reveal a delicate balance between the $\alpha$ - and the $\beta$-phase for the given experimental parameters (see calculations). The corresponding simulated STM images, displayed in Fig. 2 b and 2 e respectively, agree well with the experimental observations. 


\section{Phase transition between the two phases}

Once coexisting domains of both phases are present on the surface, we can locally and reversibly induce the phase transition by changing the bias voltage. Fig. 3 shows that the transition between the $\alpha$ - and the $\beta$-phase can be simply induced by the external electric field of the STM tip. Fig. 3 a displays three $\mathrm{Cu}(111)$ terraces covered with the $\beta$-phase of CO. c-f show the area marked in a with higher resolution. Between the images, the tip was placed at the center of the dashed frame and the bias voltage applied to the sample was ramped to $-3 \mathrm{~V}$ to switch from $\beta$ - to $\alpha$-phase (prior to $\mathrm{c}$ and e) and to $1 \mathrm{~V}$ to switch from $\alpha$ - to $\beta$-phase (prior to $\mathrm{d}$ and $\mathrm{f}$ ). This process was repeated more than 50 times. All images were acquired at a bias of $-0.5 \mathrm{~V}$, a parameter which does not alter the distribution of the phases.
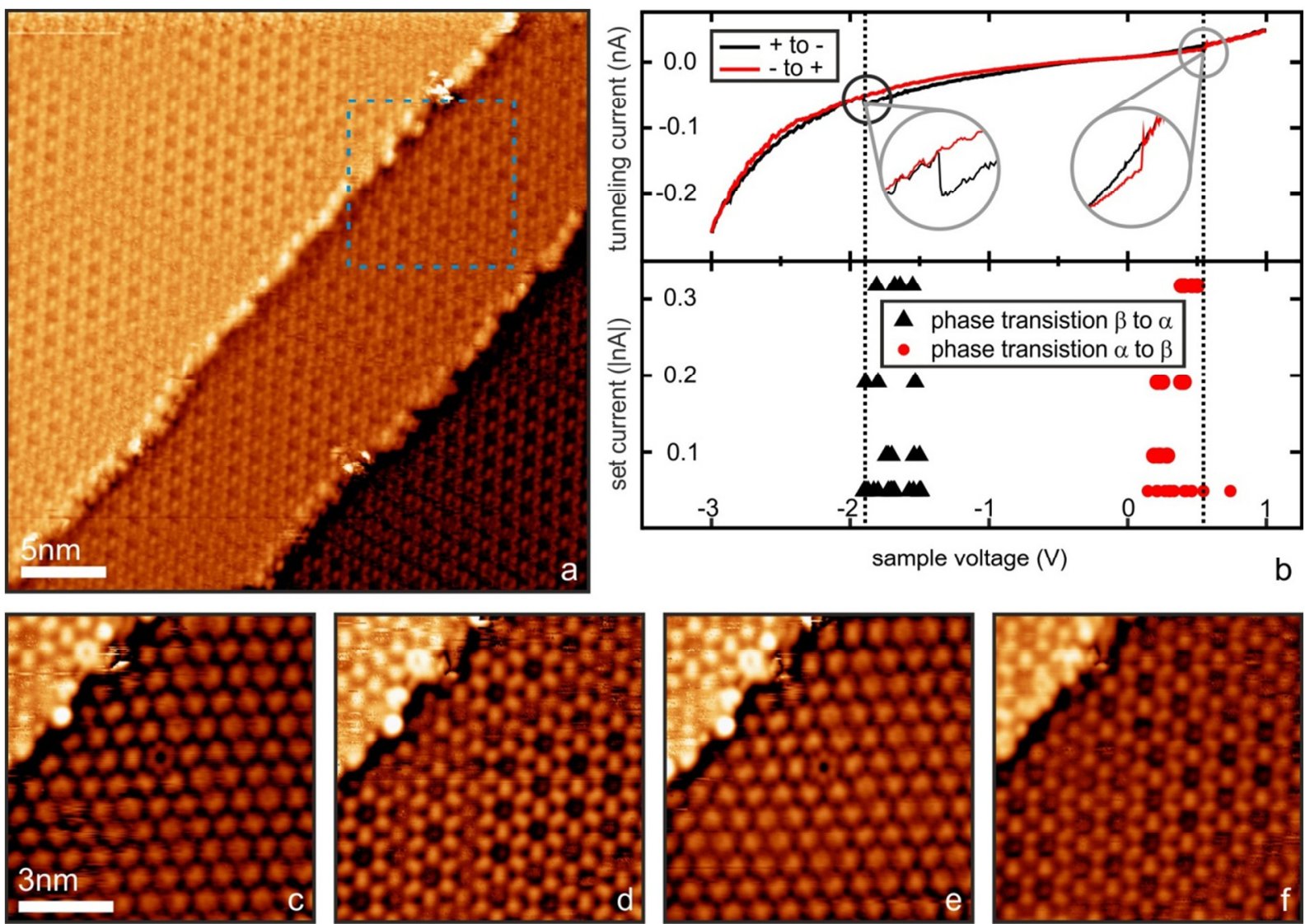

Fig. 3 Switching of the phases by STM. a displays the area prior to the manipulation. To induce the phase transition the tip was placed in the center of the dashed square and the voltage was ramped to $1 \mathrm{~V}$ and $-3 \mathrm{~V}$ respectively. $\mathrm{b}$ (upper part) tunneling current as function of sample bias. The kinks in the curve indicate the switching between the different phases. c-f show the induced phases on the part of the small terrace indicated by the dashed frame in a. 
As displayed in Fig. $3 \mathrm{~b}$, the dynamics of the process are revealed by monitoring the tunneling current while the voltage is varied. The phase transition can be identified by a discontinuity in the tunneling current marking the thresholds of the electric field required for the transition. The upper part of Fig. $3 \mathrm{~b}$ displays the tunneling current as function of bias in a range between $-3 \mathrm{~V}$ and $+1 \mathrm{~V}$; the insets provide a close up of the small kinks in the curve indicating the phase transitions. One clearly sees a hysteresis between the threshold for the formation of the $\beta$-phase going from negative to positive voltage (red) and the one for the formation of the $\alpha$ phase going from positive to negative voltage (black). This indicates a first order phase transition which requires an activation energy.

In the lower part of Fig. 3 b, the threshold values of more than 20 phase transitions are collected. The data were acquired for slightly different tip sample distances adjusted by the set current at a bias of $+1 \mathrm{~V}$. Apparently, the tunneling current has no major influence on the thresholds for the phase transition. Moreover if the tip is retracted by $30 \mathrm{~nm}$ during the bias ramp (corresponding STM images are given in appendix B Fig. 6) the phase transition can still be induced. However, at this large distance the threshold values are increased. Since the tunneling current completely vanishes at a distance of $30 \mathrm{~nm}$, we conclude that the transition is not induced by the tunneling current. This is in contrast to other experiments, observing changes to the surface structure at constant tunneling distance while the bias voltage is ramped to high values in conjunction with increased tunneling currents [28]. At this large distance, chemical short range forces are negligible as well. For the given experimental conditions, only the Coulomb forces exerted by the electric field are left.

The experiments have been repeated with tips of different shape but also different material (chromium tips as well as platinum-iridium tips). In all cases, the phase transition is induced by the electric field. However, the threshold values for the required bias voltage vary from tip to tip and also as a function of time and coverage. 


\section{Calculations}

Our calculations confirm the findings by vibrational spectroscopy about the adsorption sites of the molecules: for the $\beta$-phase all the molecules adsorb on top sites, while for the $\alpha$-phase bridge and eventually hollow sites are occupied as well. This leads to non-trivial dipole distributions on the surface. Both experimentally and theoretically, the work function for the $\alpha$-phase is about $0.1 \mathrm{eV}$ higher than for the $\beta$-phase despite the slightly higher coverage [36, 37] reflecting the complexity of the dipolar arrangement on the surface.
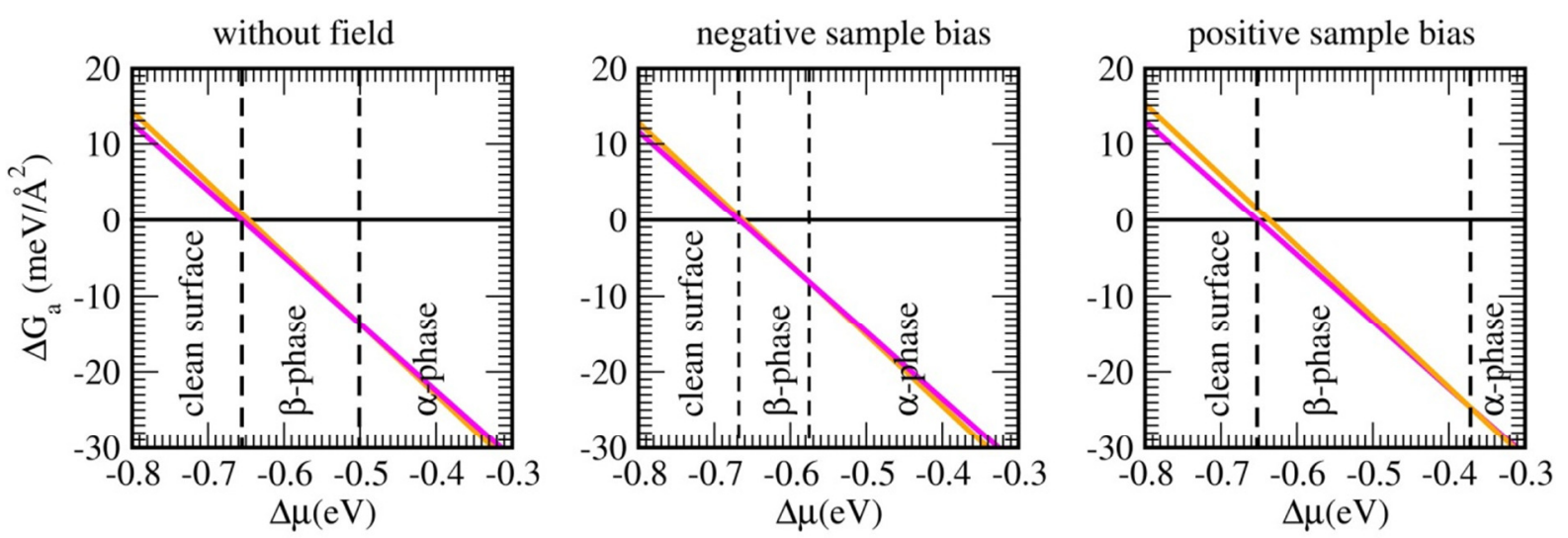

Fig. 4 Change in the Gibbs free energy due to the formation of adsorbate monolayers. The orange and the magenta lines show the results for the $\alpha$ - and the $\beta$-phase respectively. The lowest Gibbs free energy corresponds to the stable phase. The two dashed lines mark the phase transitions as a function of the reservoir's chemical potential, $\Delta \mu(\mathrm{T}, \mathrm{P})$. If a small electrical field of $0.3 \mathrm{~V} / \AA$ is applied such that the molecular dipole is more stable, the stability domain of the $\beta$-phase greatly increases. However, as soon as the field decreases, the $\alpha$-phase is favored.

Fig. 4 displays a computed phase diagram of the adsorbed phases which permits us to rationalize the effect of the external electric field on the adsorbed phase (see appendix A for details). The key finding is that the two available phases are extremely close both in mean adsorption energy per molecule and in molecular density. As a consequence, the Gibbs free energies of the two phases are very close for a wide range of the $\mathrm{CO}$ gas-phase temperatures and pressures, and a small perturbation can easily change the stable phase. In agreement with the larger work function, the $\alpha$-phase dipole is slightly larger. At the positive applied bias, this 
leads to a destabilization of roughly $10 \mathrm{meV}$ of the molecular dipoles seen as the adsorptionenergy difference between both phases. Since the destabilization is lower for the beta-phase (4 $\mathrm{meV}$ ), this small perturbation is enough to induce a sizable increase of the stabilization domain of the $\beta$-phase, so that it becomes the prevailing phase. As the field is reversed, the $\alpha$-phase becomes more stable due to its slightly larger molecular density.

\section{Details of the phase transition}

While the experiments can be repeated many times for several hours, there is a temporal evolution on the time scale of days due to a very slow but not completely negligible desorption of the $\mathrm{CO}$ molecules at the given temperature of $80 \mathrm{~K}$. Thus, within days the relative contribution of the $\beta$-phase slightly increases.

Since the coverage of $\mathrm{CO}$ molecules in the $\alpha$-phase is higher, the question arises where the redundant molecules go to after switching to the $\beta$-phase. Since the process can be reversed they apparently have to stay in close vicinity. A possible explanation is found if a larger area of the $\beta$-phase is studied after switching from the $\alpha$-phase. As can be seen in Fig. 5 as well as in appendix B Fig. 6, domain boundaries are formed within the $\beta$-phase. A close inspection reveals fluctuations above the domain boundaries in the tunneling current as well as in the registered topography. This indicates unstable areas, possibly due to mobile $\mathrm{CO}$-molecules which do not find an adsorption site with sufficient binding energy. We assume that these molecules represent the excess due to the difference in coverage of about $3 \%$. That explains why we were not able to produce larger areas of the $\beta$-phase without these domain boundaries starting from the $\alpha$-phase.

If both phases are present within the area of the surface which is studied by STM, the domain size of the $\alpha-(\beta-)$ phase increases for a negative (positive) sample bias close to the threshold 
for the transition to the $\alpha-(\beta-)$ phase. Like a 'molecular reversi' rows of ordered molecules in neighboring areas are converted from $\alpha$ to $\beta$ and vice versa. Fig. 5 a-d show how the domain boundaries between the two phases move and the $\beta$-phase expands at the expense of the $\alpha$ phase at positive sample bias. A close inspection reveals that defects on the surface, e.g. the one indicated by the arrow, can temporarily pin a domain boundary.
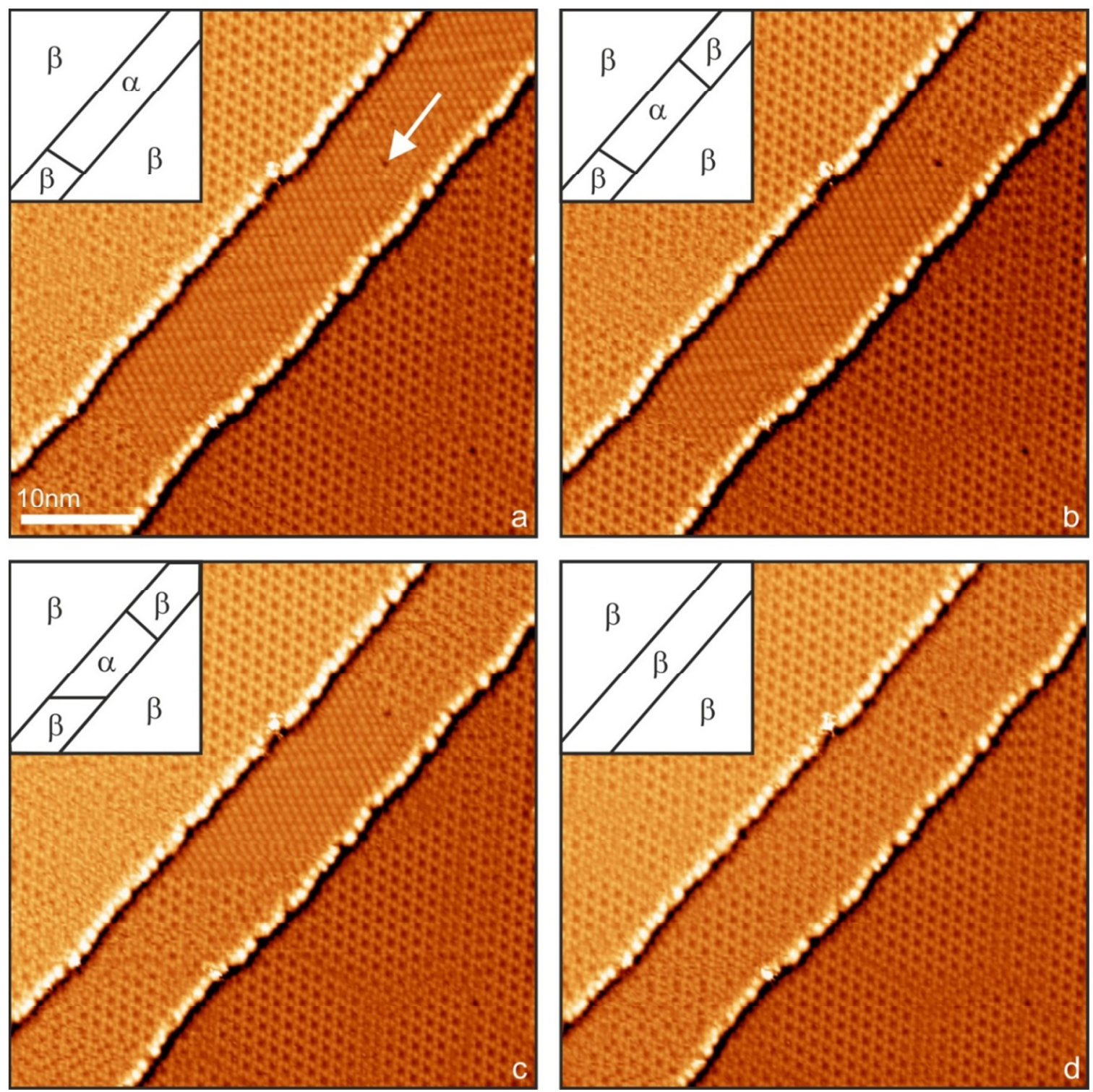

Fig. 5 Expansion of the $\beta$-phase. Prior to the measurements, a small area was transformed to the $\alpha$-phase by sweeping the bias voltage to $-1.5 \mathrm{~V}$. The sequence of STM images (a-d) reveals how the $\alpha$-phase is successively converted back to the $\beta$-phase. The measurements were done at a tunneling current of $50 \mathrm{pA}$, a bias voltage of $0.5 \mathrm{~V}$ and a time interval of 3 min. 
The hysteresis observed in Fig. $3 \mathrm{~b}$ between switching back and forth suggests that an activation energy has to be overcome to start the conversion of the phases. The relatively constant value of the threshold indicates that this energy is required for one specific initial process starting a chain reaction which continues until the reaction comes to a stop at a defect or a boundary, e.g. an atomic step. We tested this concept by analyzing the threshold as a function of the size of the transformed area and do not find any correlation. However, the hysteresis is rate dependent, i.e. the thresholds for switching depend on the dwelling time. Hence, the barrier given by the activation energy is overcome by thermal activation. If the tunneling current is measured as function of bias as shown in Fig. 3 b, the width of the hysteresis shrinks if the slew rate of the voltage is decreased. As discussed in more detail (see appendix B Fig. 8b), we use this to evaluate the impact of the electric field on the activation energy. The hysteresis of the phase transition enables us to create arbitrary pattern of patches of the two phases, which are long lived if the appropriate electric field for coexistence is used after the formation (see appendix B Fig. 9).

\section{Conclusions}

Our experiments reveal a structural 2D phase transition between two rather complex molecular arrangements of $\mathrm{CO}$ on $\mathrm{Cu}(111)$, which is reversible despite the slightly different coverages. Since the transition is efficiently triggered by the electric field of an STM tip using voltages in the range of $+/-3 \mathrm{~V}$ it opens a new field of research, especially due to the excellent control of the experimental conditions. In contrast to thermally induced transitions, very localized areas can be reversibly transformed and patterns of the two phases may be written on a small scale. Since the phases differ in their electric surface potential, well defined structures in the electric potential (field) above the surface can be designed. The switching times can be very fast since the bias can be changed rapidly (If the bias is chosen high enough, the dwelling time for the thermal activation can be reduced substantially.). In contrast 
to the common observation of phase transitions, not only macroscopic quantities of a large ensemble may be studied, but the position of every element of the system is directly accessed. Given the ease of the experiment, it is an ideal model system providing unprecedented insight into the physics of structural phase transitions on the atomic scale. Additionally, it opens an unforeseen development in the field of atomic manipulation. Future bottom-up strategies will combine atom-manipulation technics with phase transitions physics so as to generate new macroscopic structures.

\section{Acknowledgements}

We thank Dietrich Wolf for fruitful discussions and a critical reading of the manuscript. ICN2 acknowledges support from the Severo Ochoa Program (MINECO, Grant SEV-2013-0295). R.R. and N.L. acknowledge financial support from Spanish MINECO (Grant No. MAT201238318-C03-02 with joint financing by FEDER Funds from the European Union). P.A. Acknowledges the MINCyT (project PICT Bicentenario 1962), CONICET (project PIP 0272), and UNR (project PID 19/I375) and the CCT-Rosario Computational Center. C.A.B. acknowledges financial support through the German research council (DFG). 


\section{References [1-37]}

[1] A. J. Heinrich, C. P. Lutz, J. A. Gupta, D. M. Eigler, Molecule cascades. Science 298, 1381(2002).

[2] K. K. Gomes, W. Mar, W. Ko, F. Guinea, H. C. Manoharan, Designer Dirac fermions and topological phases in molecular graphene, Nature 483, 306 (2012)

[3] P. Papon, J. Leblond, P. H. E. Meijer, The Physics of Phase Transitions: Concepts and Applications (Springer Science \& Business Media, 2007).

[4] M. Gitterman, V. Halpern, Phase Transitions: A Brief Account with Modern Applications, (World Scientific, 2004) .

[5] R. V. Solé, Phase Transitions (Princeton University Press, 2011).

[6] A. A. Hyman, K. Simons, Beyond oil and water-phase transitions in cells. Science 337, 1047(2012).

[7] J. Zhang, C. Z. Chang, P. Tang, Z. Zhang, X. Feng, K. Li, L. l. Wang, X. Chen, C. Liu, W. Duan, K. He, Q. K. Xue, X. Ma, Y. Wang, Topology-driven magnetic quantum phase transition in topological insulators. Science 339, 1582(2013).

[8] K. Heinz, Diffuse low-energy electron diffraction. Prog. Surf. Sci. 27, 239(1988).

[9] J. B. Hannon, R. M. Tromp, Low-energy electron microscopy of surface phase transitions. Annu. Rev. Mater. Res. 33, 263(2003).

[10] K.Yagi, Y. Tranishiro, Studies of surface phase transition by electron microscopy. Phase Transitions: A Multinational Journal 53, 197-214(1995).

[11] C. Bai, Scanning tunneling microscopy and its applications. (Springer Verlag, New York, 2000).

[12] A. Alessandrini, P. Facci, Phase transitions in supported lipid bilayers studied by AFM. SoftMatter 10, 7145(2014).

[13] I. Brihuega, O. Custance, R. Pérez, J. M. Gómez-Rodriguez, Intrinsic Character of the $(3 \times 3)$ to $(3 \times 3)$ Phase Transition in Pb/Si (111). Phys. Rev. Lett. 94, 046101(2005).

[14] W. Srour, D.G. Trabada, J. I. Martínez, F. Flores, J. Ortega, M. Abuín, Y. FagotRevurat, B. Kierren, A. Taleb-Ibrahimi, D. Malterre, A. Tejeda, Ultrafast Atomic Diffusion Inducing a Reversible $(23 \sqrt{ } \times 23 \sqrt{ }) \mathrm{R} 30^{\circ} \leftrightarrow(3 \sqrt{ } \times 3 \sqrt{ }) \mathrm{R} 30^{\circ}$. Transition on Sn/Si(111):B, Phys. Rev. Lett. 114, 196101(2015).

[15] R. Gutzler, Th. Sirtl, J. F. Dienstmaier, K. Mahata, W. M. Heckl, M. Schmittel, M. Lackinger, Reversible Phase Transitions in Self-Assembled Monolayers at the LiquidSolid Interface: Temperature-Controlled Opening and Closing of Nanopores. J. Am. Chem. Soc. 132, 5084-5090(2010).

[16] R. Koch, M. Sturmat, High-temperature STM of the phase transitions of Au (110) and Pt (110). Surf. Sci. 861, 402-404(1998).

[17] Y.-Ch. Lin, D. O. Dumcenco, Y.-Sh. Huang, K. Suenaga, Atomic mechanism of the semiconducting-to-metallic phase transition in single-layered MoS2. Nat. Nanotechn. 9, 861(2014).

[18] Z. G. Ye, H. Schmid, Optical, dielectric and polarization studies of the electric fieldinduced phase transition in $\mathrm{Pb}(\mathrm{Mg} 1 / 3 \mathrm{Nb} 2 / 3) \mathrm{O} 3$ [PMN]. Ferroelectrics 145, 83(1993). 
[19] A. Ullah, R. A. Malik, A. Ullah, D. S. Lee, S. J. Jeong, J. S. Lee, I. W. Kim, Ch. W. Ahn, Electric-field-induced phase transition and large strain in lead-free Nb-doped BNKT-BST ceramics. Journal of the European Ceramic Society 34, 29(2014).

[20] E. M. Choi, Y. H. Yoon, S. Lee, H. Kang, Freezing transition of interfacial water at room temperature under electric fields. Phys. Rev. Lett. 95, 085701(2005).

[21] Z. Qian, G. Wei, Electric-field-induced phase transition of confined water nanofilms between two graphene sheets. J. Phys. Chem. A 2014, 118, 8922(2014).

[22] G. J. Su, L. Y. Huang, L. X. Zhang, H. J. Lu, In-situ STM observation of the phase transition of two-dimensional 2, 5-distyrylpyrazine nanostructure adsorbed on $\mathrm{Au}$ (111) in an electrochemical environment. Sci. China Chem. 56, 2013(5).

[23] F.P. Cometto, K. Kern, M. Lingenfelder, Local Conformational Switching of Supramolecular Networks at the Solid/Liquid Interface. ACS Nano 9, 55445550(2015).

[24] K.-A. Alexander, N. Duerloo, Yao Li, E. J. Reed, Structural phase transitions in twodimensional Mo-and W-dichalcogenide monolayers. Nat. Commun. 5, 4214(2014).

[25] K. Sagisaka, D. Fujita, Emergence of p (2× 2) on highly doped n-type Si (100) surfaces: A scanning tunneling microscopy and spectroscopy study. Phys. Rev. B 71, 245319(2005).

[26] Y. Pennec, M. Horn von Hoegen, X. Zhu, D. C. Fortin, M. R. Freeman, Dynamics of an Ising chain under local excitation: A scanning tunneling microscopy study of $\mathrm{Si}$ (100) dimer rows at 5 K. Phys. Rev. Lett. 96, 026102(2006).

[27] S. Morita, Y. Sugawara, Atomically resolved imaging of Si (100) $2 \times 1,2 \times 1$ : H and $1 \times$ 1: $2 \mathrm{H}$ surfaces with noncontact atomic force microscopy. Jpn. J. Appl. Phys. 41, 4857(2002).

[28] J. H. Schott, H.S. White, Electric Field Phenomena in STM: Tip Deformations and Au (111) Surface Phase Transitions During Tunneling Spectroscopy Experiments. Langmuir 9, 3471(1993).

[29] J. Zhang, J. Liu, J. Lin Huang, Ph. Kim, C. M. Lieber, Creation of Nanocrystals via a STM Tip-Induced Solid-Solid-Phase Transition. Science 274, 759(1996).

[30] S. Schintke, S. Berner, A. Alkauskas, A. Baratoff, H.-J. Güntherodt, T. A. Jung, Phase transistion of a confinded 2D molecular layer reversibly triggered by the tip of a scanning tunneling microscope. PSI scientific reports 141(2003).

[31] R. Raval, S. F. Parker, M. E. Pemble, P. Hollins, J. Pritchard, M. A. Chester, FT-rairs, eels and leed studies of the adsorption of carbon monoxide on $\mathrm{Cu}$ (111). Surf. Sci. 203, 353(1988).

[32] J. Pritchard, On the structure of CO adlayers on $\mathrm{Cu}(100)$ and $\mathrm{Cu}$ (111). Surf. Sci. 79, 231(1979).

[33] B. E. Hayden, K. Kretzschmar, A. M. Bradshaw, An infrared spectroscopic study of $\mathrm{CO}$ on $\mathrm{Cu}$ (111): The linear, bridging and physisorbed species. Surf. Science 155, 553(1985).

[34] P. Hollins, J. Pritchard, Isotopic mixing for the determination of relative coverages in overlayer structures: CO on Cu (111). Surf. Sci. 99, L389(1980). 
[35] L. Bartels, G. Meyer, K.-H. Rieder, The evolution of CO adsorption on Cu (111) as studied with bare and CO-functionalized scanning tunneling tips. Surf. Sci. 432, L621L626(1999).

[36] P. Hollins, J. Pritchard, Interactions of CO molecules adsorbed on Cu(111), Surf. Sci. 89, 486(1979).

[37] W. Kirstein, B. Krüger, F. Thieme, CO adsorption studies on pure and Ni-covered Cu (111) surfaces. Surf. Sci. 176, 505(1986). 